\title{
Ambulatório de Práticas Integrativas e Complementares: um estudo de caso sobre fitoterapia na atenção primária à saúde no município de Fortaleza-CE
}

\author{
Maria Raquel Lima Lacerda ${ }^{a^{*}}$, Kellen Miranda Sáb, Ronald Targino Nojosa ${ }^{c}$, Carine \\ Sousa dos Santos ${ }^{\mathrm{a}}$, Daniele Mary Silva de Brito ${ }^{\mathrm{a}}$
}

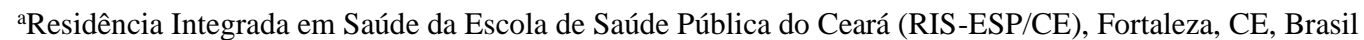

bUniversidade Estadual do Ceará, Fortaleza, CE, Brasil

'Universidade Federal do Ceará, Fortaleza, CE, Brasil

Histórico do Artigo

Recebido em:

23/07/2019

Aceito em:

$19 / 01 / 2020$

Palavras-chave: Fitoterapia; Práticas Integrativas e Complementares; Atenção Primária à Saúde

Keywords:

Phytotherapy;

Integrative and

Complementary

Practices; Primary

Health Care

\section{RESUMO}

A fitoterapia é dentre as Práticas Integrativas e Complementares de Saúde (PIC's), uma das mais utilizadas na Atenção Primária à Saúde (APS). O Núcleo Ampliado de Saúde da Família e Atenção Básica (NASF-AB) tem importante papel na promoção e utilização dessa prática. Este trabalho teve como objetivo avaliar como os usuários do Ambulatório de PIC's e profissionais de uma Unidade de Atenção Primária à Saúde (UAPS) da Regional II de Fortaleza-Ceará, perceberam a inserção da fitoterapia através de oficinas sobre uso de plantas medicinais. O estudo foi descritivo e exploratório com abordagem quantitativa e qualitativa desenvolvendo-se como estudo de caso. Foram utilizados dois questionários semiestruturados: um para os usuários e outro para profissionais. Foi realizada análise descritiva quantitativa e análise de conteúdo. Na amostra havia 40 profissionais e 12 usuários. Verificou-se que a comunidade atendida demonstrou grande interesse e aceitação pela fitoterapia e oficinas promovidas pelo Serviço de Nutrição. As plantas com maior frequência de citação foram: Camomila (Matricaria chamomilla) e Capim Santo (Cymbopogom citratus). A principal indicação das plantas medicinais utilizadas foi calmante. $O$ trabalho evidenciou que a equipe multiprofissional de saúde acha importante a fitoterapia na APS, embora perceba que não há incentivo ao uso dessa terapêutica em serviço. Conclui-se que a implantação de um ambulatório de PIC's, através do NASF-AB em apoio a Estratégia de Saúde da Família, possibilitou a inserção de uma fitoterapia cientificamente embasada, através do nutricionista. Bem como, há a necessidade de incentivos governamentais para que os profissionais de saúde da APS possam ter acesso a uma formação consolidada em PIC's.

\section{Ambulatory of Integrative and Complementary Practices: a case study on phytotherapy in primary health care in Fortaleza-CE}

\section{ABSTRACT}

Phytotherapy is among the Integrative and Complementary Health Practices (ICHP's), one of the most used in Primary Health Care (PHC). The Extended Family Health and Primary Care Center (NASF-AB) plays an important role in promoting and using this practice. This study aimed to evaluate how users of the ICHP's Ambulatory and professionals of a Primary Health Care Unit (PHCU) of the Regional II of Fortaleza-Ceará, realized the insertion of herbal medicine through workshops on the use of medicinal plants. The study was descriptive and exploratory with a quantitative and qualitative approach developing as a case study. Two semi-structured questionnaires were used: one for users and one for professionals. Descriptive quantitative analysis and content analysis were performed. In the sample there were 40 professionals and 12 users. It was found that the users attended at the outpatient clinic showed great interest and acceptance for phytotherapy and workshops promoted by the Nutrition Service. The plants with the highest citation frequency were: Chamomile (Matricaria chamomilla) and Lemongrass (Cymbopogom citratus). The main indication of the medicinal plants used was calming. The study showed that the multiprofessional health team considers phytotherapy important in PHC, although they realize that there is no incentive to use this therapy in service. It was concluded that the implementation of an ICHP's outpatient clinic, through NASF-AB in support of the Family Health Strategy, enabled the insertion of a scientifically based herbal medicine through the nutritionist. As well as, there is a need for government incentives for PHC health professionals to have access to consolidated ICHP's training.

\footnotetext{
* Autor correspondente: raquellacerda2810@gmail.com (Lacerda M.R.L.)
} 


\section{Introdução}

A Atenção Primária à Saúde (APS) é definida como o primeiro contato da população com a rede assistencial dentro do sistema de saúde, caracterizando-se, principalmente, pela continuidade e integralidade da atenção (1).

Os Núcleos Ampliados de Saúde da Família e Atenção Básica (NASF-AB), atualmente, regulamentados pela Portaria $\mathrm{n}^{\circ} 2.488$, de 21 de outubro de 2011 e, inicialmente denominados de Núcleos de Apoio à Saúde da Família, foram criados em 2008 com o objetivo de se integrarem e apoiarem às equipes de Saúde da Família e de Atenção Básica. Esses núcleos realizam diversas atividades que incluem as Práticas Integrativas e Complementares (PIC's) (1).

As PIC são baseadas em um modelo de atenção humanizada, com foco na integralidade dos indivíduos, atuando no campo de prevenção de agravos, da promoção, manutenção e recuperação da saúde. Foram institucionalizadas no Sistema Único de Saúde (SUS) brasileiro pela Portaria Gabinete do Ministro (GM)/Ministério da Saúde (MS) nº 971, de 3 de maio de 2006, através da Política Nacional de Práticas Integrativas e Complementares (PNPIC), a qual define diretrizes sobre a oferta de serviços (2).

Em março de 2017, a PNPIC foi ampliada em 14 outras práticas a partir da publicação da Portaria GM n ${ }^{\circ}$ 849/2017 sendo consolidada através da Portaria GM do Ministério da Saúde $N^{\circ} 2$ de 28 de setembro de 2017(3). Em 2018, a Portaria $N^{\circ}$ 702, de março de 2018, alterou a Portaria de Consolidação $\mathrm{n}^{\circ}$ 2/GM/MS/17, para incluir novas práticas totalizando vinte e nove PIC's.

Dentre as PIC's utilizadas no SUS e na APS, destaca-se a fitoterapia uma terapêutica caracterizada pelo uso de plantas medicinais em suas diferentes formas farmacêuticas, sem a utilização de substâncias ativas isoladas, ainda que de origem vegetal (2). A Política Nacional de Plantas Medicinais e Fitoterápicos (PNPMF) e o Programa Nacional de Plantas Medicinais descrevem o uso, as diretrizes, delineiam ações e o papel dos profissionais de saúde relacionados à essa Prática Integrativa e Complementar (PIC) (4).

Com o intuito de propiciar a utilização das PIC's no cuidado aos pacientes de uma Unidade de Atenção Primária à Saúde (UAPS) de Fortaleza-Ceará, foi criado em 2018, um Ambulatório de PIC's em um espaço comunitário na área adstrita ao posto de saúde da UAPS, onde através do serviço de Nutrição, foram realizadas oficinas de orientação sobre o uso de plantas medicinais através de círculos de cultura (6) com os usuários do serviço.

Dentre os profissionais de saúde aptos a conduzir ações relacionadas à fitoterapia está o nutricionista, por meio da Resolução n ${ }^{\circ}$ 525/2013 do Conselho Federal de Nutricionistas, alterada pela Resolução ${ }^{\circ} 556 / 2015$, que regulamentou a prática da Fitoterapia para 0 profissional como complemento da prescrição dietética (5). Podendo desenvolver atividades relacionadas às indicações de plantas medicinais, suas preparações, e formas de consumo, considerando todos os determinantes de saúde das populações, além de promover educação continuada dos Agentes Comunitários de Saúde (ACS) sobre a temática (7).

Partindo da hipótese que a fitoterapia tem ampla aceitação, promove o cuidado integral, prevenção de agravos, promoção, manutenção e recuperação da saúde dos indivíduos (2), a pesquisa procurou avaliar como os usuários do Ambulatório de PIC's e profissionais de uma UAPS da Regional II de Fortaleza-Ceará, perceberam a inserção da fitoterapia através de oficinas sobre uso de plantas medicinais. 


\section{Materiais e métodos}

Trata-se de um estudo descritivo e exploratório com abordagem quantitativa e qualitativa como estudo de caso (8). Realizou-se nos dados quantitativos a análise descritiva quantitativa e nos subjetivos realizou-se tratamento qualitativo tendo como base a análise de conteúdo de Bardin (9) e Minayo (10). A análise de conteúdo constitui uma metodologia de pesquisa usada para descrever e interpretar o conteúdo de toda classe de documentos e textos. Essa análise, conduzindo a descrições sistemáticas, qualitativas ou quantitativas, ajuda a reinterpretar as mensagens e a atingir uma compreensão de seus significados num nível que vai além de uma leitura comum (11).

A coleta de dados foi realizada de acordo com Lakatos; Marconi (12) por meio de questionários semiestruturados, sendo um para os usuários do ambulatório e outro para profissionais. Os instrumentos da coleta de dados continham questões objetivas de múltipla escolha e perguntas diretas com respostas subjetivas.

Os dados qualitativos do estudo foram divididos em duas partes temáticas: "Aceitação de Plantas Medicinais e Fitoterápicos" (fitoterapia como alternativa de cuidado, uso subaproveitado e incentivo e apoio ao uso da fitoterapia) e "Feedback sobre as oficinas do Ambulatório de PIC's (conhecimentos adquiridos, opiniões e sugestões).

A pesquisa foi realizada entre os meses de dezembro de 2018 a março de 2019 e o local foi uma UAPS da Secretaria Executiva Regional (SER) II em Fortaleza-Ceará. A SER II de Fortaleza é formada por 20 bairros, onde habitam 334.868 pessoas, correspondendo a $13,50 \%$ da população da capital e possui área de 44,42 km² (13).O ambulatório de PIC's da UAPS foi utilizado para aplicação dos instrumentos de coleta de dados, para isso, funcionando uma vez por semana, durante o período de coleta de dados da pesquisa.

As populações-alvo da pesquisa consistiram em: (a) usuários encaminhados pelos profissionais de saúde da UAPS ao Ambulatório de PIC's e (b) profissionais da unidade de saúde. Foram convidados a participar da pesquisa os usuários que compareceram ao Ambulatório de PIC's, no período de abril de 2018 a dezembro de 2018 e o critério para inclusão foi assiduidade mínima em dois encontros no período. Quanto aos profissionais da saúde, foram incluídos na pesquisa aqueles que constavam no Atesto Municipal de Funcionamento da UAPS de 2019, totalizando 44 profissionais prescritores ou orientadores do uso de plantas medicinais. Estes caracterizaram-se por três equipes da Estratégia Saúde da Família (ESF), formadas por: Agentes Comunitários de Saúde (ACS), médico, enfermeiro, técnico de enfermagem, dentista, técnico ou auxiliar de saúde bucal e uma equipe multiprofissional de residentes formada por médico, enfermeiro, dentista, psicólogo e fisioterapeuta. Devido às recusas e desistências ao estudo chegou-se a uma amostra de 40 profissionais e 12 usuários do serviço.

Após a inclusão, todos os usuários do ambulatório de PIC's passaram por um processo de avaliação e acompanhamento semanal realizado pelo nutricionista. De acordo com a Resolução $n^{\circ}$ 525/2013 do Conselho Federal de Nutricionistas, alterada pela Resolução $\mathrm{n}^{\circ} 556 / 2015$ (5), dentre os profissionais de saúde aptos a conduzir ações relacionadas à fitoterapia está o nutricionista. A evolução dos usuários foi registrada em ficha de acompanhamento padronizada. Os círculos de cultura (2) foram realizados mensalmente e a pergunta geradora utilizada foi: "O que você sabe sobre plantas medicinais?". As dúvidas e questionamentos provenientes dos círculos de cultura (2) foram utilizadas para montar as oficinas práticas com preparações de infusões e lambedores caseiros seguindo as preparações descritas por Matos (14).

O artigo seguiu as orientações da resolução 466/12 do Conselho Nacional de Saúde (CNS), tendo o projeto de pesquisa sido apreciado e aprovado pelo Comitê de Ética da Escola de Saúde Pública do Ceará - ESP/ CE com número de CAAE 
99761418.9.0000.5037.

\section{Resultados e discussão}

\subsection{Perfil da amostra}

Neste estudo a amostra constitui-se de 12 usuários do Ambulatório de PIC's e de 40 profissionais da unidade de saúde. Os entrevistados em sua maioria foram mulheres (Tabela 1), o que corrobora com os dados obtidos por Gadelha et. al (15) onde foi avaliado o uso de fitoterápicos e de plantas medicinais na saúde básica do município de Sousa-PB, concluindo-se que o público feminino tem mais interesse pelo assunto. Houve uma prevalência de adultos em relação aos idosos (Tabela 1) e a amostra de profissionais apresentou média de idade de 42,5 anos, mínimo 25 e máximo de 68 anos. A variabilidade das idades, medida pelo desvio padrão, foi de 9,9 anos. O perfil etário dos entrevistados demonstrou apenas um respondente do sexo masculino, 46 anos, os outros onze respondentes foram do sexo feminino, com idade mínima de 46, máximo 67 e média de 55 anos. Esses resultados assemelham-se ao estudo realizado por Zeni et al. (16), que encontraram uma porcentagem de usuários atendidos na Atenção Primária de Blumenau (SC) de 73,6\% com faixa etária entre 20 a 64 anos.

Tabela 1 - Caracterização sociodemográfica dos entrevistados.

\begin{tabular}{|c|c|c|c|c|}
\hline \multicolumn{3}{|c|}{ Variável } & $\mathrm{N}$ & $\%$ \\
\hline \multicolumn{5}{|c|}{$\operatorname{Sexo}(n=52)$} \\
\hline & & & 45 & 86,5 \\
\hline & & & 7 & 13,5 \\
\hline \multicolumn{5}{|c|}{ Faixa etária anos $(\mathrm{n}=52)$} \\
\hline 25 & a menos de & 35 & 8 & 15 \\
\hline 35 & a menos de & 45 & 12 & 23 \\
\hline 45 & a menos de & 55 & 23 & 44 \\
\hline 55 & a menos de & 65 & 7 & 14 \\
\hline 65 & a menos de & 75 & 2 & 4 \\
\hline
\end{tabular}

No grupo de usuários do Ambulatório de PIC's, 6 (50\%) participantes possuíam grau de instrução de Ensino Fundamental Incompleto e 11 (92\%) indicaram renda entre um e dois salários mínimos. Esses dados coincidem com os resultados de um estudo realizado sobre o perfil da demanda atendida em UAPS's do Sul e Nordeste do Brasil (14), no qual $36,3 \%$ indicou grau de instrução semelhante ao encontrado no grupo de usuários do Ambulatório de PIC's. Porém, entra em conflito com Araújo et. al (15) que encontrou maior parte de sua população com renda entre dois e três salários mínimos.

Estudo segundo o Ipea (18) os indicadores sociais tornaram-se instrumentos indispensáveis na definição de ações para implementação de políticas públicas que visam ao estabelecimento de processos de superação das desigualdades sociais. Nesse sentido, a promoção de ações em PIC's com foco na qualidade de vida pode se constituir em importante instrumento para os NASF-AB. Denota-se a importância da fitoterapia defendida por Matos (14), das plantas medicinais como opções de recursos terapêuticos, acessíveis e de baixo custo, conforme preconiza a Política Nacional de Plantas Medicinais e Fitoterápicos do Ministério da Saúde.

Dos 40 profissionais da UAPS que participaram da pesquisa, 17 são ACS's, uma técnica em saúde bucal, duas auxiliares em saúde bucal, quatro técnicas de enfermagem, seis enfermeiras, uma médica na função de gestora da unidade, outras três médicas da ESF e quatro dentistas. Entre os profissionais que compõem a equipe NASF-AB, apenas um 
psicólogo, uma fisioterapeuta, ambos residentes da Rede de Residências Integradas em Saúde da Escola de Saúde Pública do Ceará (ESP-CE). Quando comparadas a equipe da ESF versus equipe do NASF-AB da unidade pesquisada, verificou-se uma distribuição irregular das especialidades de saúde. Essa irregularidade é evidenciada em duas pesquisas, Matos et. al. (19) e Santos, Léda e Oliveira (20), que tiveram em suas amostras um predomínio de enfermeiros na ESF, porém difere dos resultados obtidos no presente estudo, no qual foi encontrado uma predominância de ACS's.

O predomínio de ACS's está de acordo com a Política Nacional de Atenção Básica (21), demonstrando a importância desse profissional para a abordagem e orientações domiciliares sobre o uso das plantas medicinais cientificamente validadas em preparações caseiras, bem como, sobre a necessidade do seu envolvimento em trabalhos que busquem conhecer o consumo de fitoterápicos e plantas medicinais nas comunidades.

A distribuição irregular das especialidades de saúde quando comparadas equipe da ESF versus equipe do NASF-AB na UABS foi um dado preocupante, visto que o NASF-AB da unidade foi de suma importância para a inserção das PIC's e não contou, no período da pesquisa, com profissionais fixos da UABS, somente residentes temporários. Esses dados confirmam o que é asseverado por Sá et al. (22) em relação à consolidação da fitoterapia no SUS, onde se faz necessária a intervenção do poder público no sentido de proporcionar políticas públicas de financiamento que permitam a manutenção e perpetuação de profissionais e serviços. A situação evidenciada no estudo corrobora para que os serviços permaneçam em moldes que favorecem o uso do medicamento sintético (22) em detrimento das PIC's. Reforça-se que os objetivos de um NASF-AB são: apoiar, ampliar, aperfeiçoar a atenção e a gestão da saúde na Atenção Básica/Saúde da Família (1).

\subsection{Uso das plantas medicinais}

A PNPMF, de 2006, e o Programa Nacional de Plantas Medicinais e Fitoterápicos, de 2008, proporcionaram um aumento expressivo no número de programas de fitoterapia no SUS (23). É possível observar o reflexo desse aumento no presente estudo, pois a maioria dos profissionais entrevistados orientou e/ou prescreveu plantas medicinais e/ou fitoterápicos o que é reforçado por Sá et al. (22) onde é citado que a maioria dos conselhos de profissionais de saúde regulamenta a fitoterapia no exercício profissional, o que colabora para sua expansão, visto que a resistência de alguns profissionais de saúde em trabalhar com plantas medicinais é diretamente relacionada ao desconhecimento do assunto.

Em relação aos usuários do Ambulatório de PIC's todos usam plantas medicinais no seu cuidado em casa, o que revela a importância da implantação do serviço na ABS como mecanismo para alcance de uma prática segura.

A Tabela 2 evidencia uma prevalência de indicação/prescrição de plantas medicinais calmantes, hipotensoras leves e digestivas em detrimento da prescrição de plantas medicinais e fitoterápicos para o aparelho musculoesquelético. Ressalta-se que a UABS tem foco nesse atendimento. Assim, salienta-se a necessidade de um trabalho de difusão da informação no que concerne à assistência farmacêutica para a UABS com vistas a possibilitar a prescrição dos recursos terapêuticos disponíveis visto que tanto a Relação Nacional de Medicamentos Essenciais (24) disponibiliza fitoterápicos para essa indicação como o Programa Farmácia Viva, instituído pelo Ministério da Saúde no âmbito do Sistema Único de Saúde, mediante a Portaria MS/GM no 886/2010 (25).

A planta utilizada com mais frequência entre os usuários (camomila) não correspondeu à planta mais prescrita e/ou orientada pelos profissionais capim santo (Cymbopogon 
citratus) (Tabela 2). Também apareceram citadas uma vez pelos usuários: alecrim (Lippia origanoides), babosa (Aloe vera), canela de velho (Miconia albicans), colônia (Alpinia zerumbeti), jatobá (Hymenaea courbaril) e um dos respondentes não especificou a planta que utiliza. Dentre as plantas citadas a canela de velho (Miconia albicans) ainda não apresenta segurança e eficácia comprovada cientificamente, e não possui registro na Agência Nacional de Vigilância Sanitária (ANVISA) sendo proibida a fabricação, distribuição, divulgação, comercialização e uso (26), fato que reforça a importância da orientação realizada pelo Ambulatório de PIC's.

Tabela 2 - Plantas medicinais e/ou fitoterápicos usados pelos usuários do Ambulatório de PIC e prescritos e/ou orientados pelos profissionais de uma UAPS de Fortaleza - CE.

\begin{tabular}{lclc}
\hline \multicolumn{1}{c}{ Usuários } & & \multicolumn{2}{c}{ Profissionais } \\
\hline Camomila & Frequência & Nome popular ou fitoterápicos ${ }^{\circledR}$ & Frequência \\
Erva cidreira & 6 & Capim santo & 11 \\
Capim santo & 5 & Erva cidreira & 8 \\
Boldo & 4 & Boldo & 6 \\
Erva doce & 3 & Camomila & 6 \\
Hortelã & 3 & Não especificou & 5 \\
Malvariço & 2 & Erva doce & 4 \\
& 2 & Eucalipto & 4 \\
& & Canela & 3 \\
& & Ginkgo biloba & 3 \\
& & Valeriane & \\
& & Alfavaca & 3 \\
& & Gengibre & 2 \\
& & Hortelã & 2 \\
Total & & Lambedor de abacaxi & 2 \\
& & Lambedor de romã & 2 \\
& & Passiflora & \\
& & Total & 2 \\
\hline
\end{tabular}

Os profissionais citaram também: aroeira (Myracrodruon urudeuva), babosa (Aloe vera), cebolinha, chá de hortelã (Mentha sp), chás (não especificou a planta), cumaru (Dipteryx odorata), lambedores (não especificou a planta), macela (Achyrocline satureioides), olho da goiaba (Psidium guajava) com pitanga (Eugenia uniflora), pomada de aroeira (Myracrodruon urudeuva), quebra pedra (Phyllanthus niruri), Sintocalmy ${ }^{\circledR}$, suco de chuchu (Sechium edule), sumo de capim santo (Cymbopogon citratus), xarope de chambá (Justicia pectoralis), xarope de cumaru (Dipteryx odorata), xarope de guaco (Mikania glomerata).

$\mathrm{Na}$ Tabela 3 observa-se a finalidade das plantas usadas pelos usuários e profissionais do Ambulatório de PIC's, prevalecendo a indicação calmante. Outras finalidades foram: acne, alívio de dores, anti-inflamatório, cicatrização, colecistite, congestão nasal, crises de garganta, dores articulares, expectorante, gripe, queimaduras, tontura, vômito e zumbido. 
Tabela 3 - Finalidade das plantas usadas pelos usuários e profissionais do Ambulatório de PIC, Fortaleza - CE.

\begin{tabular}{lclc}
\hline \multicolumn{1}{c}{ Usuários } & & \multicolumn{2}{c}{ Profissionais } \\
\hline Finalidade & Frequência & \multicolumn{1}{c}{ Finalidade } & Frequência \\
\hline Calmante & 14 & Não especificadas & 20 \\
Parte da alimentação & 4 & Calmante & 14 \\
Dispepsia & 4 & Insônia & 8 \\
Insônia & 3 & Dispepsia & 7 \\
Tosse & 2 & Tosse & 6 \\
Confecção de lambedor & 2 & Resfriado & 5 \\
Dores reumáticas & 1 & Ansiedade & 3 \\
Hipertensão arterial & 1 & Diarreia & 3 \\
Memória & 1 & Hipertensão arterial & 2 \\
& & Infecções de vias aéreas & 2 \\
Hidratação do cabelo & 1 & superiores & \\
Esteatose hepática & 1 & & 70 \\
Total & 34 & Total & \\
\hline
\end{tabular}

Percebeu-se que a maioria dos profissionais $(n=21)$ da unidade relatou não haver incentivo do próprio serviço em relação ao trabalho com plantas medicinais e fitoterápicos o que é confirmado por Sá et al. (22) que a qualificação dos profissionais de saúde na área de plantas medicinais deve estar atrelada a ações primárias na esfera municipal de financiamentos específicos para o setor.

Os usuários do serviço avaliaram em quase totalidade $(\mathrm{n}=11)$ as oficinas com plantas medicinais como boas ou ótimas, considerando a importância de novos aprendizados. Zeni et al. (16) assevera as plantas medicinais como alternativa terapêutica de grande aceitação pela comunidade com a necessidade de garantia do acesso, bem como profissionais qualificados capazes de fornecer orientações sobre sua utilização.

Os dados qualitativos do estudo foram divididos em duas partes temáticas: "Aceitação de Plantas Medicinais e Fitoterápicos" (fitoterapia como alternativa de cuidado, uso subaproveitado e incentivo e apoio ao uso da fitoterapia) e "Feedback sobre as oficinas do Ambulatório de PIC's (conhecimentos adquiridos, opiniões e sugestões) foram analisados e transcritos ipsis litteris verificando-se que todos os profissionais de saúde demonstraram respostas positivas em relação ao uso de plantas medicinais e fitoterápicos:

“Ótimo, ajuda no tratamento." (profissional n 1)

"Bom, pois com um chazinho se resolve uma pessoa com ansiedade" (profissional $\left.\mathrm{n}^{\circ} 39\right)$

"Ótimo, ajuda a reduzir o uso de antibiótico e anti-inflamatórios" (profissional $\mathrm{n}^{\circ} 20$ )

Os profissionais consideraram a fitoterapia como adjuvante ao tratamento de doenças, alternativa aos medicamentos alopáticos (para pacientes alérgicos, antibióticos e antiinflamatórios), para recuperação da saúde e evitar superlotação das UAPS. Esses resultados corroboram com Nóbrega et al. (27) que assevera a fitoterapia como importante terapêutica no apoio ao tratamento de doenças e infecções, com seus efeitos comprovados cientificamente. Também foi relatado pelo grupo que apesar da fitoterapia ser tão segura e eficaz quanto os medicamentos alopáticos, e mais acessível à população (em relação ao custo financeiro), ainda existe um subaproveitamento dessa terapêutica no posto de saúde e o seu uso deve ser incentivado para profissionais e comunidade, sendo implantado como terapia complementar.

"Os fitoterápicos são mais baratos, mais seguros e tão eficazes quanto remédios de "farmácias"'” (profissional n 35) 


\begin{abstract}
"Minha sugestão seria que a fitoterapia fosse disseminada e trabalhada constantemente junto à comunidade" (profissional n ${ }^{\circ} 37$ )

"Há muitos anos trabalhávamos na Unidade com pomada de aroeira, xarope de cumaru etc; sendo que não foi mais dispensado pela farmácia do município." (profissional $\mathrm{n}^{\circ} 8$ )

"Incentivado por outras pessoas, raramente. Mas sinto entusiasmo interno/próprio" (profissional $\mathrm{n}^{\circ} 13$ )

"Sim, já fui incentivada por alguns profissionais para esse uso em favor da melhora do problema" (profissional n²9)
\end{abstract}

Pode-se inferir que esse subaproveitamento pode ter relação com a falta de incentivo ao treinamento em serviço dos profissionais e também por deficiências advindas da própria graduação $(19,22)$.

A análise quantitativa da frequência de profissionais que se sentem incentivados e motivados a utilizarem a fitoterapia, recebeu justificativas de ordem qualitativa, onde foram ressaltados: entusiasmo próprio, entusiasmo através das oficinas de fitoterapia do ambulatório na figura da nutricionista responsável pelas atividades, incentivo por outros profissionais, entusiasmo após a consolidação das PIC's no SUS e Residência Integrada em Saúde.

Verificou-se que a Rede de Residências Integradas em Saúde da Escola de Saúde Pública do Ceará (ESP-CE) possibilitada pelo Plano Municipal Educação Permanente de Saúde do município de Fortaleza bem como a preceptoria (profissionais do serviço que acompanham os residentes) foram importantes para a criação do ambulatório de PIC dentro da APS o que possibilitou as oficinas (28), dando incentivo aos profissionais a conhecerem e indicarem a fitoterapia.

Em relação ao grupo dos usuários do Ambulatório de PIC's da UAPS foram verificados os seguintes conhecimentos adquiridos: confecção de lambedor, chás, alcoolatura e sabonete líquido de alecrim pimenta, ervas estimulantes e relaxantes. O que reforça a necessidade do compartilhamento de conhecimentos e práticas, bem como alternativas terapêuticas autônomas, interação entre o usuário do SUS, a equipe de saúde e a proteção da biodiversidade e das riquezas naturais $(29,30)$. Outras atividades e práticas abordadas nos grupos foram: massoterapia, acupuntura, auriculoterapia, como fazer escalda pés, relaxamento em grupo, como montar um jardim com material reciclado e como fazer adubo caseiro:

"Aprendeu sobre os chás das ervas para que serve cada um. Aprendeu sobre ervas estimulantes e relaxantes" (usuária $n^{\circ} 4$ )

"Relaxamento como trabalhar em grupo" (usuária n ${ }^{\circ}$ )

"Massagem, cuputura, erico; pena que poderia ter mais muito mais" (usuária $n^{\circ} 2$ )

A maioria dos profissionais relatou benefícios com a existência de oficinas sobre fitoterapia, dentre eles estão: aquisição de novos conhecimentos para profissionais e comunidade, fortalecimento da cultura popular, importante na APS e maior acessibilidade da população mais carente a essa terapêutica, o que é reforçado pela PNPMF sobre as plantas medicinais serem acessíveis a população, resgatarem o conhecimento popular e favorecerem a participação popular, entre outros benefícios (31). Apenas dois profissionais não conheciam a existência dessas oficinas.

"Acho que são importantes e enriquecem a prática dos profissionais de saúde" (profissional $n^{\circ} 6$ )

"Importante $\mathrm{p} /$ fortalecer o conhecimento popular e disseminar o conteúdo" (profissional $\mathrm{n}^{\circ} 19$ ) 
"Que ajudam bastante principalmente a população mais carente" (profissional n²5)

Alguns participantes da pesquisa sugeriram diferentes assuntos para as oficinas como preparações caseiras, temas específicos (plantas e temas), oficinas direcionadas aos profissionais, a continuidade do ambulatório.

"Sobre unha de gato" (usuária n 3)

"Fitoterapia no tratamento de doenças crônicas" (profissional n ${ }^{\circ} 15$ )

"Fortalecer o uso de fitoterápicos para insônia e ansiedade visto que se trata de problema muito presente na fala dos usuários dessa UAPS" (profissional $\mathrm{n}^{\circ} 8$ )

"Que os cursos fossem direcionados a todos os profissionais..." (profissional n ${ }^{\circ} 17$ )

"Apenas que continue a existir e continue ensinando como utilizar a fitoterapia" (profissional $\mathrm{n}^{\circ} 31$ )

Todas as sugestões fornecidas pelo grupo avaliado no estudo foram organizadas e separadas em duas partes: Preparações caseiras e Assuntos específicos (Tabela 4).

Tabela 4 - Sugestões dos participantes para as oficinas de fitoterapia.

\begin{tabular}{ll}
\hline \multicolumn{1}{c}{ Preparações caseiras } & \multicolumn{1}{c}{ Assuntos específicos } \\
\hline Preparação de lambedores & Unha de gato, Babosa, Mentrasto \\
Mel e pomadas & Fitoterapia na saúde bucal (higienização) \\
Receita para manchas na pele & Fitoterapia nas doenças crônicas \\
Fazer novamente sabonete líquido de Alecrim pimenta & Fitoterápicos para insônia e ansiedade \\
Chás e suas utilizações & Matruz, Corama (tosse) \\
Fitoterápicos e suas utilizações & Como reconhecer as plantas, colher, plantar e \\
& registrar sua eficácia \\
Inalações (alfavaca e eucalipto) & Cursos para profissionais \\
Estudo com pacientes alérgicos & Importância do uso do fitoterápico na APS \\
Xarope para gripe & Fazer cartilha para população sobre \\
& fitoterapia \\
\hline
\end{tabular}

Verificou-se que na UABS estudada há demanda para oficinas de preparação, capacitações teóricas, bem como inserção de instrumentos escritos sobre fitoterapia.

\section{Considerações finais}

A implantação de um ambulatório de PIC's, através do Núcleo Ampliado de Saúde da Família e Atenção Básica em apoio a Estratégia de Saúde da Família, possibilitou a inserção de uma fitoterapia cientificamente embasada, através de um profissional de saúde habilitado, nesse caso específico, o nutricionista.

Na população estudada prevaleceu o público feminino e se concluiu que esse público tem mais interesse pelos assuntos relacionados à saúde e PIC's, ressaltando a necessidade de campanhas que utilizem uma abordagem mais direcionada ao público masculino, como forma de atrair e propiciar qualidade de vida e saúde preventiva na ABS.

O estudo revelou que a fitoterapia tem ampla aceitação e é uma importante prática para a promoção da qualidade de vida e saúde da população. Entretanto, para que isso seja efetivado se faz necessário a garantia do acesso, bem como profissionais de saúde qualificados, visto que a fitoterapia ainda é pouco utilizada na maioria das UAPS, em parte pela dificuldade na oferta.

Há a necessidade de incentivos governamentais para que os profissionais de saúde da APS possam ter acesso a uma formação consolidada em PIC's como forma de ampliar as opções terapêuticas disponíveis em apoio às atividades da ESF. Entre os principais incentivos que devem ser viabilizados estão: liberação do serviço para capacitação, 
treinamento em serviço, oferta permanente dos insumos necessários à prática das PIC's. A parceria entre instituições voltadas ao ensino e pesquisa, no caso do estudo, a Rede de Residências Integradas em Saúde da Escola de Saúde Pública do Ceará (ESP-CE) mostrou-se ferramenta importante para ampliar a oferta de profissionais no NASF-AB. Entretanto, ressalta-se que tal ferramenta não deve substituir a contratação de profissionais de saúde permanentes, visto que sua oferta é de cunho temporário, o que afeta a oferta dos serviços.

\section{Referências}

1. Brasil. Ministério da Saúde. Secretaria de Atenção à Saúde. Departamento de Atenção Básica. Núcleo de Apoio à Saúde da Família/Ministério da Saúde, Secretaria de Atenção à Saúde, Departamento de Atenção Básica. - Brasília: Ministério da Saúde, 2014. 116 p.: il. - (Cadernos de Atenção Básica, n. 39).

2. Brasil. Ministério da Saúde. Secretaria de Atenção à Saúde. Departamento de Atenção Básica. Política nacional de práticas integrativas e complementares no SUS: atitude de ampliação de acesso/Ministério da Saúde. Secretaria de Atenção à Saúde. Departamento de Atenção Básica. - 2. ed. - Brasília: Ministério da Saúde, 2015. 96 p.: il.

3. Brasil. Ministério da Saúde. Portaria $\mathrm{n}^{\circ} 28$ de setembro de 2017. Consolidação das normas sobre as políticas nacionais de saúde do Sistema Único de Saúde. Diário Oficial da União, 28 set.

4. Brasil. Ministério da Saúde. Secretaria de Ciência, Tecnologia e Insumos Estratégicos. Departamento de Assistência Farmacêutica. Política e Programa Nacional de Plantas Medicinais e Fitoterápicos/Ministério da Saúde, Secretaria de Ciência, Tecnologia e Insumos Estratégicos, Departamento de Assistência Farmacêutica. - Brasília: Ministério da Saúde, 2016. 190 p.

5. Brasil. Conselho Federal de Nutricionistas. Resolução $\mathrm{n}^{\circ}$ 556, de 11 de abril de 2015. Altera as Resoluções no 416, de 2008, e n 525, de 2013, e acrescenta disposições à regulamentação da prática da Fitoterapia para o nutricionista como complemento da prescrição dietética. Diário Oficial da União, 14 mai.

6. Freire P. Educação como prática de liberdade. Rio de Janeiro: Paz e Terra; 1991.

7. Brasil. Ministério da Saúde. Secretaria de Atenção à Saúde. Departamento de Atenção Básica. Práticas integrativas e complementares: plantas medicinais e fitoterapia na Atenção Básica/Ministério da Saúde. Secretaria de Atenção à Saúde. Departamento de Atenção Básica. - Brasília: Ministério da Saúde, 2012. 156 p.: il. - (Série A. Normas e Manuais Técnicos) (Cadernos de Atenção Básica; n. 31).

8. Yin RK. Estudo de caso: planejamento e métodos. Porto Alegre: Bookman; 2001.

9. Bardin, L. Análise de conteúdo. Lisboa: Edições 70; 1988.

10. Minayo, MCS. O desafio do conhecimento: pesquisa qualitativa em saúde. São Paulo: HucitecAbrasco; 1998.

11. Moraes R. Análise de conteúdo. Educação, Porto Alegre 1999; 22 (37): 7-32.

12. Lakatos EM, Marconi MA. Técnicas de pesquisa: planejamento e execução de pesquisas, amostragens e técnicas de pesquisa e elaboração, análise e interpretação de dados. São Paulo: Atlas; 2008.

13. Prefeitura de Fortaleza. [Internet]. Ceará, 2019. Disponível em: https://www.fortaleza.ce.gov.br/institucional/a-secretaria-316

14. Matos FJA. Plantas medicinais: guia de seleção e emprego das plantas usadas em fitoterapia no Nordeste do Brasil. Fortaleza: Imprensa Universitária; 2007.

15. Gadelha CS, Junior VMP, Bezerra KKS, Maracajá PB, Martins DSS. Utilização de medicamentos fitoterápicos e plantas medicinais em diferentes segmentos da sociedade. Revista Verde (Pombal - PB - Brasil) 2015; 10 (3): 01-15.

16. Zeni ALB, Parisotto AV, Mattos G, Helena ETS. Utilização de plantas medicinais como remédio caseiro na Atenção Primária em Blumenau, Santa Catarina, Brasil. Ciência \& Saúde Coletiva 2017; 22(8): 2703-2712.

17. Araújo CRF, Silva AB, Tavares EC, Costa EP, Mariz SR. Perfil e prevalência de uso de plantas medicinais em uma unidade básica de saúde da família em Campina Grande, Paraíba, Brasil. Revista 
de Ciências Farmacêuticas Básica e Aplicada 2014; 35 (2):233-238.

18. Instituto de Pesquisa Econômica Aplicada. Atlas da vulnerabilidade social nos municípios brasileiros. [Internet]. Brasília, 2015. Disponível em: http://portalms.saude.gov.br/acoes-e-programas/saude-dafamília/nucleo-de-apoio-a-saude-da-familia-nasf.

19. Mattos G, Camargo A, Sousa CA, Zeni ALB. Plantas medicinais e fitoterápicos na Atenção Primária em Saúde: percepção dos profissionais. Ciência \& Saúde Coletiva 2018; 23(11): 3735-3744.

20. Santos SS, Léda PHO, Oliveira DR. Plantas Medicinais e Fitoterapia em Oriximiná - Pará, Brasil: Percepção e Intenção de Uso pelos Profissionais do Sistema Único de Saúde (SUS). Vittalle - Revista de Ciências da Saúde 2018; 30 (1): 11-25.

21. Brasil. Ministério da Saúde. Portaria GM/MS n 2.488, de 21 de outubro de 2011. Aprova a Política Nacional de Atenção Básica, estabelecendo a revisão de diretrizes e normas para a organização da Atenção Básica, para a Estratégia Saúde da Família (ESF) e o Programa de Agentes Comunitários de Saúde (PACS). Diário Oficial da União 2011; 21 out.

22. Sá K, Lima A., Bandeira M, Andriola W, Nojosa R. Avaliando o impacto da política brasileira de plantas medicinais e fitoterápicos na formação superior na área de saúde. Revista Ibero-Americana de Estudos em Educação 2018; 13(4): 1106-1131.

23. Luis HLR. Análise dos programas de plantas medicinais e fitoterápicos no Sistema Único de Saúde (SUS) sob a perspectiva territorial. Ciência \& Saúde Coletiva 2019; 24(5): 1733-1742.

24. Brasil. Ministério da Saúde. Secretaria de Ciência, Tecnologia e Insumos Estratégicos. Departamento de Assistência Farmacêutica e Insumos Estratégicos. Relação Nacional de Medicamentos Essenciais: RENAME 2018 [recurso eletrônico] / Ministério da Saúde, Secretaria de Ciência, Tecnologia e Insumos Estratégicos, Departamento de Assistência Farmacêutica e Insumos Estratégicos. - Brasília: Ministério da Saúde, 2018. 218 p.

25. Brasil. Ministério da Saúde. Portaria GM/MS nº 886, de 20 de abril de 2010. Institui a Farmácia Viva no âmbito do Sistema Único de Saúde (SUS). Diário Oficial da União 2010; 22 abr.

26. Brasil. Agência de Vigilância Sanitária. Resolução-RE nº 400, de 16 de fevereiro de 2017. O Diretor da Agência Nacional de Vigilância Sanitária, no uso das atribuições que lhe conferem o art. 151, V e VI, e o art. 54, I, $\S 1^{\circ}$ do Regimento Interno aprovado nos termos do Anexo I da Resolução da Diretoria Colegiada - RDC $n^{\circ}$ 61, de 3 de fevereiro de 2016, e a Resolução da Diretoria Colegiada - RDC $n^{\circ} 99$, de 02 de agosto de 2016. Diário Oficial da União, 16 fev.

27. Nóbrega AL, Ugulino PTD, Cajá DF, Dantas AEF. A importância da orientação dos profissionais das equipes de saúde da família acerca do uso da fitoterapia. Revista Brasileira de Educação e Saúde 2017; 7 (1): 43-48.

28. Ceará. Secretaria Municipal da Saúde de Fortaleza. Plano Municipal de Educação Permanente em Saúde 2018 - 2021 [recurso eletrônico] / Secretaria Municipal da Saúde de Fortaleza. - Fortaleza: Secretaria Municipal da Saúde, 2018. 98 p. il.

29. Antonio GD, Tesser CD, Moretti-Pires, RO. Fitoterapia na atenção primária à saúde. Revista de Saúde Pública 2014; 48 (3): 541-553.

30. Antonio GD, Tesser CD, Moretti-Pires, RO. Contribuições das plantas medicinais para o cuidado e a promoção da saúde na atenção primária. Interface (Botucatu) 2013; 17 (46): 615-33.

31. Figueredo CA, Gurgel IGD, Junior GDG. A Política Nacional de Plantas Medicinais e Fitoterápicos: construção, perspectivas e desafios. Physis Revista de Saúde Coletiva 2014; 24 (2): 381-400.

32. Werkema MCC. Ferramentas estatísticas básicas para o gerenciamento de processos. Belo Horizonte: Eletrônica; 1996. 